\title{
Introduction to Deep Brain Stimulation
}

\author{
Andres M. Lozano, MD, PhD, ${ }^{1}$ and Robert E. Gross, MD, PhD² \\ 'Division of Neurosurgery, University of Toronto, Ontario, Canada; and \\ 2Department of Neurosurgery, Emory University, Atlanta, Georgia
}

It is estimated that over 160,000 patients worldwide have received deep brain stimulation (DBS) to date predominantly for Parkinson's disease and other movement disorders. With the success of this therapy, a greater appreciation of the clinical benefits and adverse effects is being realized. Neurosurgeons are increasingly paying attention to the technical details of these procedures and optimizing targeting, surgical techniques, and programming to improve outcomes.

In this issue, the nuances of surgical techniques for DBS are covered by Dr. House. Dr. Toda et al. and Mr. Chartrain et al. tackle the approach to treating tremors, either essential tremor or Holmes tremor, using either a single target or, in cases of difficult-to-treat tremors, using more than one target and interleaving the stimulation. These abstracts and videos will be appreciated by both those who are being initiated to DBS and the more seasoned practitioners who are looking for helpful hints to tackle challenging cases.

KEYWORDS deep brain stimulation; tremor; movement disorders; video 